\title{
Conocimientos, actitudes y prácticas de los principios bioéticos del profesional de enfermería en el cuidado del paciente hospitalizado, del Hospital II De Vitarte-EsSalud, 2011
}

\author{
Liria Oportuna BARRENECHEA BACA*
}

Asistencia integral de salud-servicios geriátricos S.A.C, Avenida Aviación, 2410 - 3er Piso Of.176 - San Borja - Lima

\begin{abstract}
RESUMEN
El objetivo de la investigación fue determinar el nivel de conocimientos, actitudes y prácticas de los principios bioéticos del profesional de enfermería en el cuidado del paciente hospitalizado, del Hospital II de Vitarte-EsSalud, 2011. Estudio de diseño cuantitativo, de alcance exploratorio y descriptivo. Se realizó en una muestra conformada por 35 profesionales de enfermería que laboran en el área asistencial de los Servicios de Medicina, Cirugía, Emergencia y UVI (Unidad de Vigilancia Intensiva). Se utilizó un cuestionario para medir el nivel de conocimientos, una escala para actitudes y una guía de observación para prácticas. Las áreas comprendidas en este estudio fueron: Deontología, Ética, Bioética, Beneficencia, Autonomía, Secreto Profesional, Consentimiento Informado, Justicia. Los resultados encontrados fueron que $20 \%$ de los participantes posee un nivel excelente de conocimientos sobre los principios bioéticos, el $45.7 \%$ tiene un nivel bueno, el $31.4 \%$ regular y el $2.9 \%$ tiene un nivel deficiente. Con respecto a los niveles de actitud de los principios bioéticos el $71.4 \%$ es positiva y el $28.6 \%$ presenta un nivel de actitud medianamente positiva. En cuanto a las prácticas, el $60 \%$ de los participantes practica los principios bioéticos durante el cuidado al paciente. Lo que significa que la mayoría de los profesionales de enfermería presenta un nivel de conocimientos y un nivel de actitudes de los principios bioéticos, bueno y positivo respectivamente. Asimismo, el nivel de prácticas es muy aceptable y requerido en el cuidado del paciente.
\end{abstract}

Palabras clave: Bioética, cuidado del paciente, conocimientos bioéticos.

\begin{abstract}
The objective of the research was to determine the level of knowledge, attitudes and practices of bioethical principles of nursing in inpatient care, the Hospital II-EsSalud Vitarte, 2011. Design Studio quantitative, exploratory and descriptive scope. Was conducted in a sample composed of 35 nurses who work in nursing care Medical Services, Surgery, Emergency and ICU (Intensive Care Unit). A questionnaire was used to measure the level of knowledge, attitudes and a scale for a guide to practical observation. Areas covered in this study were: Ethics, Ethics, Bioethics, beneficence, autonomy, confidentiality, informed consent, justice. The results were that $20 \%$ of participants have an excellent level of knowledge of bioethical principles, $45.7 \%$ have a good level, $31.4 \%$ and $2.9 \%$ regularly has a poor level. Regarding attitude levels of bioethical principles is positive $71.4 \%$ and $28.6 \%$ has a moderately positive attitude level. As for practices, $60 \%$ of participants practiced bioethical principles during patient care. Which means that most nurses, have a level of knowledge and level of attitudes of bioethical principles, good and positive respectively. Similarly, the level of practice is acceptable and required in patient care.
\end{abstract}

Keywords: Bioethics, patient care, bioethical knowledge.

*Correspondencia: liria@upeu.edu.pe, 51-1 -980467650 


\section{INTRODUCCIÓN}

La ética del cuidado no solo obliga aliviar el sufrimiento, sino también a mejorar dentro de lo posible la calidad de vida del paciente, la clave es centrarse en el enfermo como individuo, considerarlo como un ser único, con derecho a obtener la verdad amablemente, a decidir por sí mismo y a recibir excelencia en el cuidado físico y psicoespiritual, todo dentro del marco de los cuatro principios fundamentales de la bioética: Autonomía, justicia, no maleficencia y beneficencia (Taboada, 1998).

Por ello es importante saber si los profesionales de salud conocen los principios bioéticos que rigen cada una de las etapas fundamentales durante el cuidado y tratamiento en el proceso de la enfermedad y la forma adecuada para aplicarse estos principios, pues todo razonamiento ético que se realiza debe pasar por el reconocimiento de la dignidad de la persona y de los derechos que le son inherentes.

Entre las causas principales asociadas a la ausencia de los principios bioéticos en el cuidado del paciente, son el desconocimiento y el déficit de actualización con respecto a temas éticos de la especialidad.

Porra, Díaz y Cordero (2001), en una investigación realizada sobre "Conocimientos y aplicación de los principios éticos y bioéticos en el proceso de atención de enfermería”, comprobaron que los principios éticos y bioéticos más conocidos por las enfermeras fueron, el respeto a las personas, el secreto profesional y la justicia; mientras que los de beneficencia y autonomía son los menos reconocidos, aunque en la práctica diaria todos son aplicados de forma aceptable. Los autores también refieren que los profesionales de salud que más dominan los principios éticos y bioéticos fueron las licenciadas en enfermería, y aquellas que han participado en cursos de ética y bioética.

Por otro lado, Baltazar y García (2009), realizaron el siguiente trabajo de investigación, "Nivel de conocimientos bioéticos en el personal de enfermería de un hospital del sureste de Veracruz", el estudio fue descriptivo de corte transversal, con el propósito de identificar el nivel de conocimientos bioéticos en el personal de enfermería de una institución de salud. La muestra estuvo constituida por 181 enfermeras (os). Los resultados mostraron que el principio bioético más conocido por las enfermeras fue el de autonomía con un $80.7 \%$, así como el principio de beneficencia con un $78.5 \%$. En general, el $56.3 \%$ de los encuestados presenta un nivel de conocimiento regular.

Sin embargo, la existencia de problemas relacionados con el actuar profesional de la enfermera demuestra que no basta tomar en cuenta los aspectos cognoscitivos sino que es imprescindible acompañar este análisis de una valoración de la conducta moral del profesional de salud. Amaro (2004), al referirse a la conducta moral, afirma que ésta implica las actitudes, las cualidades del carácter y el sistema de valores de cada profesional de salud. Pero las cualidades del carácter no son innatas, sino que se forman en el proceso de enseñanza-aprendizaje, permitiendo el desarrollo de las habilidades necesarias para enfrentar la toma de decisiones correctas ante los conflictos morales surgidos en la práctica profesional.

Por tal motivo, el profesional de salud puede, en el transcurso de su carrera, llegar a poseer actitudes positivas o negativas que trasciendan en una toma de decisiones éticas correctas o incorrectas en su desempeño laboral.

Por otro lado, Poblete y Valenzuela (2007), en una investigación sobre el "Cuidado humanizado: un desafío para las enfermeras en los servicios hospitalarios”, encontró que los profesionales de enfermería, como una forma de sentirse valorados han relacionado el cuidado con el accionar cotidiano, considerando la tecnología como poder, orientando muchas veces su práctica hacia lo técnico y distanciándose, por tanto, de los principios morales.

Es por ello que Amaro, Marrero, Valencia, Casas y Moynelo (1996), afirman que el énfasis exagerado en la tecnología, lleva imperceptiblemente a la deshumanización. El cuidado no solo requiere que la enfermera sea científica, académica y clínica, sino también un agente humanitario y moral, como copartícipe en las transacciones de cuidados humanos.

De no ser abordado el problema, los factores antes mencionados pueden degradar la atención de enfermería, que sin lugar a dudas debe estar basada en la reciprocidad y debe poseer una calidad única y auténtica. Así lo señala el artículo 23 del Código de Ética y Deontología del Perú del Colegio de Enfermeros del Perú (2005), que la enfermera debe tener en cuenta los principios de Beneficencia y no maleficencia para tomar la decisión más adecuada y oportuna en el cuidado de la persona cuando su vida está en riesgo-

De ahí se concluye que no basta conocer, reconocer y aceptar los principios éticos que se han establecido en las relaciones sociales y profesionales para el éxito del cuidar, tampoco podrá hablarse de servicios de excelencia si no se parte de una medición objetiva de sus niveles de calidad y de un cumplimiento irrestricto de los principios éticos, en nuestra profesión: la más hu- 
mana de todas. La actuación del cumplimiento del deber de los trabajadores de la medicina solamente puede responder a los principios éticos de la profesión, fuera de cuyo marco es inconcebible la acción humanitaria (Pérez, 1999).

Durante las prácticas preprofesionales en los servicios de hospitalización, se ha observado que muchos de los pacientes, manifiestan sentimientos de molestia e incomodidad frente a la actitud del profesional de enfermería durante el cuidado y/o la realización de procedimientos. El registro de "Satisfacción del Usuario", muestra algunas opiniones referidas por los pacientes: "Un día le pedí a la enfermera que me alcance la chata, y me dijo: le pondré de sobrenombre chata. Espera que venga la técnica", "la enfermera no tiene paciencia, se enoja por gusto, me culpó de que yo manipulé la vía, y cuando le reclamé, su trato empeoró”, “fui operada en el primer turno, en la noche cuando solicité una chata, me la dieron, solicité ayuda y ésta me fue negada, diciéndome que debía valerme por mí misma, al retirar la chata se me derramó la orina mojando toda la cama. La enfermera me increpó con un gesto feo y me dijo: ya sabía que esto iba a pasar. Por favor tengan paciencia con nosotros, en esta situación necesitamos apoyo, confianza en que son buenas personas".

Por estas razones, se crea la necesidad de investigar el nivel de conocimientos, la predisposición de practicar los principios bioéticos y la aplicación de los mismos en el cuidado de Enfermería, por lo que es imprescindible propiciar la reflexión del profesional de enfermería en torno a los principios bioéticos en el ejercicio de la profesión, enfatizando la necesidad de reconocer al paciente como un sujeto autónomo que tiene derecho a saber, opinar y decidir sobre la conducta que pretenden asumir los profesionales de la salud; con la intención de promoverle su salud, evitarle enfermedades, diagnosticar, tratar y rehabilitar en caso necesario; y tener en cuenta a la persona, a la familia y a la comunidad. El objetivo del estudio es determinar el nivel de conocimientos, actitudes y prácticas de los principios bioéticos del profesional de enfermería en el cuidado del paciente hospitalizado, del Hospital II de Vitarte-EsSalud.

\section{MATERIAL Y MÉTODOS}

Estudio cuantitativo, de diseño no experimental, de corte transversal y de alcance exploratorio-descriptivo. La muestra estuvo constituida por 35 enfermeras (os) que laboran en el área asistencial de los servicios de Medicina, Cirugía, Emergencia y Unidad de Vigilancia Intensiva (UVI), del Hospital II de Vitarte-EsSalud previamente seleccionados según criterios de inclusión: Enfermeras (os) que cumplen labor asistencial en los servicios de Cirugía, Medicina, Emergencia y UVI, a pacientes hospitalizados.

Cuestionario para medir el nivel de conocimientos. Para la elaboración de este cuestionario se ha hecho adaptaciones respectivas al instrumento elaborado por Rodríguez (2003). El cuestionario contempla las siguientes dimensiones:conocimiento del principio bioético de la Beneficencia, de la no Maleficencia, Autonomía y Justicia.

Escala de actitudes. Para la elaboración de este instrumento se hizo adaptaciones respectivas al instrumento elaborado por Ospina, De Jesús, Aristizábal y Ramírez (2003), que mide el nivel de actitudes de los profesionales de enfermería en el cuidado de la salud. Donde determinada pregunta se contestó con base en las categorías de: Totalmente de acuerdo (TA); De acuerdo (DA); Indiferente (IN); En desacuerdo (ED); Totalmente en desacuerdo (TD). Donde se calificó el resultado de afirmaciones analizadas en el continuo del 1 al 5, 21 afirmaciones en un total de 101 puntos, categorizada en actitudes positivas y negativas.

Guía de observación. La guía de observación se elaboró a partir de los procedimientos correspondientes a la investigación señalados en la base teórica, siendo adaptada de la guía de observación elaborada por Remuzgo (2003). Para ponderar los ítems se ha utilizado la metodología empleada por Ortega y Suárez (2009), en el "Programa de Evaluación de la Calidad de los Servicios de Enfermería”. Para las prácticas se consideró un total de 68 puntos como máximo. 


\section{RESULTADOS}

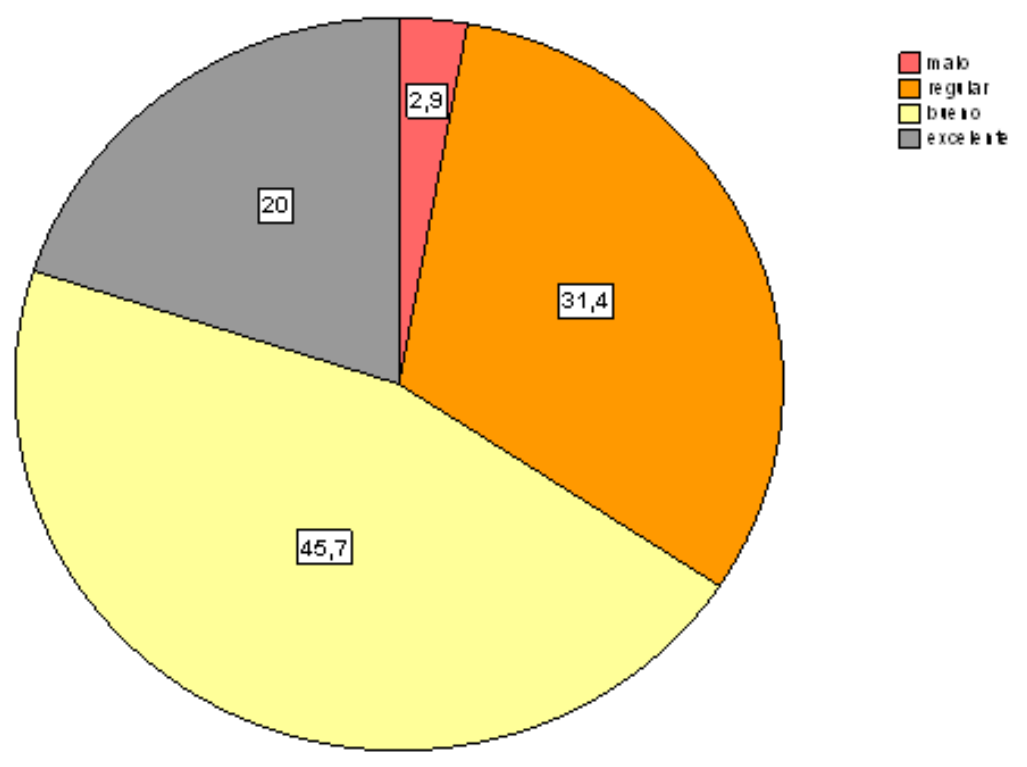

\section{Figura 1}

Nivel de conocimientos de los principios bioéticos de los profesionales de enfermería en el cuidado del paciente hospitalizado del Hospital II de Vitarte-EsSalud.

En la figura 1, se observa que el $45.7 \%$ de los profesionales de enfermería posee nivel bueno sobre conocimientos de los principios bioéticos, 31 , 4\% nivel regular y solo un $20 \%$ nivel excelente.

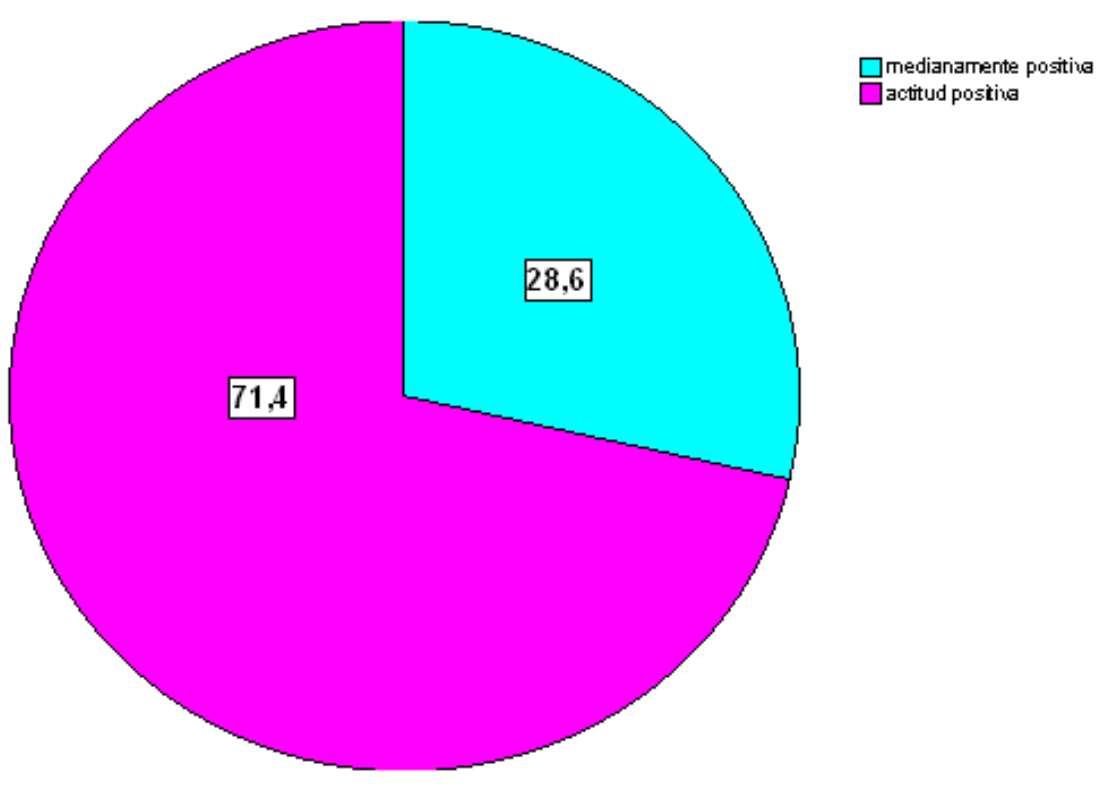

\section{Figura 2}

Nivel de actitudes hacia los principios bioéticos de los profesionales de enfermería en el cuidado del paciente hospitalizado del Hospital II de Vitarte-EsSalud.

En la figura 2, se observa que el 71.4\% de los profesionales de enfermería posee una actitud positiva hacia los principios bioéticos y un 28.6\% tiene actitud medianamente positiva. 
Tabla 1

Nivel de práctica de los principios bioéticos de los profesionales de enfermería en el cuidado del paciente hospitalizado del Hospital II de Vitarte-EsSalud según dimensiones.

\begin{tabular}{ccccccccccc}
\hline \multirow{2}{*}{ Dimensiones } & \multicolumn{4}{c}{ No practica } & \multicolumn{9}{c}{ Sí practica } \\
& \multicolumn{2}{c}{ Nunca } & \multicolumn{2}{c}{ Casi } & \multicolumn{2}{c}{ A veces } & \multicolumn{2}{c}{$\begin{array}{c}\text { Casi } \\
\text { nunca }\end{array}$} & \multicolumn{2}{c}{ Siempre } \\
\cline { 2 - 12 } & $\mathrm{N}$ & $\%$ & $\mathrm{~N}$ & $\%$ & $\mathrm{~N}$ & $\%$ & $\mathrm{~N}$ & $\%$ & $\mathrm{~N}$ & $\%$ \\
Beneficencia & 0 & 0 & 4 & 11.4 & 9 & 25.7 & 12 & 34.3 & 10 & 28.6 \\
No maleficencia & 1 & 2.9 & 7 & 20 & 12 & 34.3 & 9 & 25.7 & 6 & 17.1 \\
Autonomía & 5 & 14.3 & 6 & 17.1 & 14 & 40 & 6 & 17.1 & 4 & 11.4 \\
Justicia & 0 & 0 & 1 & 2.9 & 2 & 5.7 & 8 & 22.9 & 24 & 68.6 \\
\hline
\end{tabular}

La tabla 1 muestra que el $62.9 \%$ del personal de enfermería practica el principio de beneficencia casi siempre y siempre, el $42.8 \%$ practica casi siempre y siempre el principio de No maleficencia, y el $91.5 \%$ del personal de enfermería practica casi siempre y siempre la justicia durante el cuidado del paciente. Sin embargo, un 17.2\% no practica los principios bioéticos de no Maleficencia y Justicia.

\section{DISCUSIÓN}

Los resultados obtenidos en cuanto a los conocimientos de bioética en el profesional de enfermería, permitieron afirmar que las enfermeras (os) se evidencian tener conocimientos y esto está repercutiendo, positivamente, en la calidad de la atención de enfermería. De ahí se desprende que realicen cada proceso con mayor eficacia, como por ejemplo: los diagnósticos de enfermería, identificación de las necesidades, proyección correcta de las acciones independientes y la busca de soluciones para resolver, de forma general, los problemas de sus pacientes y, además, el respeto a él y a sus familiares como individuos.

Los resultados del estudio revelan que el principio bioético de autonomía es el más conocido por el profesional de enfermería representado por el 45.7\%, y el menos conocido es el principio de beneficencia con 51.4\%, datos que tienen similitudes y diferencias con los resultados obtenidos en el trabajo realizado por Porra, Díaz y Cordero (2001), sobre "Conocimientos y aplicación de los principios éticos y bioéticos en el proceso de atención de enfermería”, en una población de 120 enfermeras. Los resultados mostraron que el $87.5 \%$ de las licenciadas posee buenos conocimientos y el $12.5 \%$ tiene deficientes conocimientos acerca de los principios éticos y bioéticos. Según su calificación profesional, muestran que el $77.5 \%$ de las licenciadas conocía sobre el principio de autonomía, el $87.5 \%$ sobre beneficencia y el $78.3 \%$ sobre justicia.

Por lo tanto, en términos generales los profesionales de enfermería poseen un nivel bueno de conocimientos de los principios bioéticos; sin embargo, se observa que todavía poseen un conocimiento básico de lo que es, en realidad, cada uno de los principios bioéticos; por lo tanto, la preparación ética constituye una necesidad de primer orden, junto al compromiso social y al hecho de que prevalezca el sentido humanista en las acciones de salud. En los últimos años se ha producido un auge en la divulgación de los conocimientos sobre bioética a escala mundial, y llama poderosamente la atención cómo las enfermeras se interesan cada vez más por estos problemas en el interés de mejorar la atención al paciente y mejorar su calidad de vida (García, 2007). En cuanto al nivel de actitudes, el profesional de enfermería presenta en su mayoría una actitud positiva hacia principios bioéticos en el cuidado del paciente. Yagüe y García (2004), señalan que las actitudes son rasgos estables que influyen en el juicio de la persona y la acción, aun sin poseer medios o recursos necesarios para el cuidado del paciente, poseer actitudes positivas es un eje estable para el profesional de enfermería, actitudes que incidirán positivamente en la práctica profesional y diaria, en el sentido de brindar una asistencia total y humanizada, del paciente.

En el trabajo realizado por Porra, Díaz y Cordero (2001), sobre "Conocimientos y aplicación de los principios éticos y bioéticos en el proceso de atención de enfermería”, los resultados mostraron que al relacionar actitud ante los problemas y la correcta aplicación de los principios se encontró que el 55,8 \% mantenía la calma y actuaba enérgicamente aplicando correctamente los principios bioéticos, de las que refirieron poseer un carácter explosivo pero actuaban con autocontrol, el 26,6 \% aplicó correctamente los principios, mientras que las de carácter explosivo con poco autocontrol, solo el 3,3\% los aplicó correctamente.

Aristóteles menciona, que aunque las personas tengan 
diferentes rasgos de carácter, todos tenemos la capacidad para aprender o cultivar actitudes que son importantes para la moral (Durant citado por Burkhardt \& Nathaniel, 2002).

Según Bermejo (1999), muchas veces un proceso de humanización ni un proceso de estructuras, de mecanismos, no es tanto un problema de recursos, puesto que todos sabemos que se puede avanzar en un proceso de humanización con los recursos actuales, pero con actitudes distintas. Se ha dicho también que no se avanzará en este proceso a base de incorporar reglamentos, sino precisamente a través de un proceso de cambio de actitudes y de voluntad de todos los que conforman el equipo de salud, de manera que se mejore la situación actual.

La investigación demuestra que durante el cuidado del paciente, los profesionales de enfermería sí practican los principios bioéticos, datos que coinciden con un estudio realizado por Luna, Valiente, Espinosa y Goderich (2004), sobre el "Cumplimiento de los principios fundamentales de la bioética por el personal de enfermería en el área de cirugía del Hospital clínico-quirúrgico: Dr. Ambrosio Grillo Portuondo, de Santiago de Cuba", siendo la población 113 enfermeras (os). Se obtuvo los siguientes resultados: los principios bioéticos de autonomía, beneficencia y justicia entre otros, fueron cumplidos en un $100 \%$ y se respetaron los derechos del paciente, sus decisiones, preferencias y se evitaron daños, esto revela que el personal de enfermería en su trabajo diario revisa constantemente sus valores personales, los principios de la ética (beneficencia, no maleficencia, autonomía, y justicia), los derechos del paciente, sus decisiones y preferencias, evitarle daño, manipulaciones y las intervenciones y tratamientos, así como también debe rendir cuentas por la calidad del servicio prestado.

En cuanto al nivel de prácticas, los revelan que el 62.9\% del personal de enfermería practica el principio de beneficencia, el $42.8 \%$ practica el principio de no maleficencia, el $28.5 \%$ practica siempre el principio de autonomía y el $91.5 \%$ del personal de enfermería practica siempre la justicia, durante el cuidado del paciente y un $17.2 \%$ no practica los principios bioéticos. Mostrando que la mayoría de los profesionales de enfermería practica, en general, los cuatro principios bioéticos durante el cuidado del paciente. Estos resultados se asemejan a los de Porra, Díaz y Cordero (2001), que dieron a conocer en una investigación cómo las enfermeras de varios centros hospitalarios cumplían con los principios éticos y bioéticos establecidos, el 99,1 \% actuaron acorde con el principio de beneficencia; el 67,5\% al de justicia, el $100 \%$ al de autonomía. Esto quiere decir que en la práctica diaria todos los principios bioéticos son aplica- dos de forma aceptable.

Sin embargo en el presente estudio el principio de autonomía es el menos practicado a diferencia del resultado mencionado en la investigación realizada por Porra, Díaz y Cordero, 2001. Ante esta situación Miscán y Salazar (2009), afirman que la autonomía es uno de los principios bioéticos más vulnerados por los profesionales de la salud en la práctica clínica. Los autores mencionan que, a finales del año 2005, la UNESCO propone una declaración que integra la bioética y los derechos humanos. Sin embargo, a pesar de las innovaciones médicas, normas y declaraciones en pro de la protección a la persona, no se ha logrado respetar a los pacientes en toda la dimensión del ejercicio de su autonomía; lejos de ello, cada vez es más frecuente pasar el límite entre el cuidado integral y el mero cumplimiento con los procesos de atención, por ser estos parte de la actividad diaria. Por lo tanto, se observa que no es suficiente la implementación de normas, reglamentos o medidas, sino que debe haber un claro compromiso personal por el respeto de estas normas y principios bioéticos y, además, una actitud moral que brinde un cuidado humanitario.

Es fundamental remarcar que el paciente tiene derecho a ser respetado y a recibir información sobre su estado de salud, de manera que pueda ser comprendida, tomando en cuenta que excepcionalmente, se puede denegar información al paciente, cuando se considere que el conocerla puede poner en peligro su salud o su vida. No obstante, el personal de salud debe involucrar al paciente en la decisión y brindarle explicación de acuerdo con su capacidad. La autonomía es la capacidad que tiene todo ser humano de tomar decisiones y determinar aquello que más le conviene, hace mención al concepto de libertad personal, a la voluntad y a que se le respete sus derechos, sin interferencias que pretendan controlarlo y sin limitaciones personales, como lo sería una comprensión inadecuada (Fernández citado por Miscán y Salazar, 2009 ).

De acuerdo con esta explicación, la persona decide conscientemente y accede a tal o cual tratamiento. Bajo este contexto el profesional de la salud emplea el consentimiento informado (CI), para obtener la autorización de la persona en la realización de cualquier procedimiento; naturalmente, como se mencionó antes, el profesional de la salud debe explicar detalladamente y en un lenguaje sencillo, lo que implica dicho tratamiento, asegurándose de que la persona haya comprendido dicha explicación. Por tal razón, el CI es la herramienta principal para que se dé una óptima relación entre el paciente y su médico, con un trato digno, humanitario y educado para el paciente, es además una forma de favorecer la autonomía de la persona (Ramos, Beas, 
Suro, Vasquez, Machuca, De Alba, Becerra citados por Miscán y Salazar, 2009).

Otra investigación realizada por Rodríguez (2003), titulada "Exploración y aplicación de conocimientos bioéticos en el personal de enfermería”, en donde aplicó una encuesta anónima al $30 \%$ del personal de enfermería del municipio Trinidad. Se revisaron 50 procesos de atención de enfermería para evaluar en qué medida se aplican estos conocimientos en dicho proceso. Los resultados mostraron, de forma general, dificultades en los conocimientos teóricos, sin embargo, en la evaluación de la aplicación práctica de los principios de bioética en las etapas del proceso de atención de enfermería, el $80 \%$ de los encuestados aplica correctamente los principios bioéticos. De igual manera, el presente trabajo de investigación revela que a pesar de que teóricamente no se dominen a cabalidad los cuatro principios bioéticos, los resultados muestran que en su mayoría realmente éstos se aplican y se cumplen.

Se entiende que en esta actuación están implícitos patrones morales establecidos por la ética social, desarrollados desde edades tempranas como cualidades humanas que se manifiestan en nuestro modo de actuar y de pensar (Porra, Díaz y Cordero, 2001).

Por lo tanto, la comprensión del cuidado de enfermería implica la reflexión del profesional sobre su práctica, su transformación de acuerdo con los cambios en el contexto socio-político, económico y cultural y con los retos y desafíos que tiene que enfrentar para dimensionar el cuidado en los nuevos escenarios que debe compartir con otras disciplinas y sectores de la sociedad. En este sentido, es necesario recalcar que las nuevas concepciones en cuidados de enfermería están orientadas a favorecer la independencia y la autonomía de la persona, pro- moviendo una calidad de vida saludable (Ramió, 2005).

El profesional de enfermería, en el campo del cuidado al paciente, requiere de principios reguladores, de conocimientos, actitudes que le permitan aportar significativamente a la transformación del cuidado y de los servicios de salud y de enfermería (Ospina, et al. 2003). La esencia humanizante del cuidado se encuentra en ser competente en el conocimiento, en la práctica y en la actitud de cuidar (Colliere, 1993 \& Waldow, 2008).

\section{CONCLUSIONES}

- $\quad$ El $45.7 \%$ de los profesionales de enfermería presentó un nivel bueno de conocimiento acerca de los principios bioéticos.

- $\quad$ El 71.4\% de los profesionales de enfermería, presentó una actitud positiva frente a los principios bioéticos.

- El 60\% de los profesionales de enfermería sí practica los principios bioéticos durante el cuidado al paciente.

- El principio bioético más conocido por los profesionales de enfermería es el de autonomía, representado por el $45.7 \%$, y el menos conocido el principio de beneficencia con el 51.4\%.

- Los profesionales de enfermería presentaron actitud positiva frente a los cuatro principios bioéticos, siendo el principio de beneficencia el más aceptado representado por el 91.4\%.

- El principio bioético que se practica frecuentemente durante el cuidado del paciente es el de justicia, representado por el $91.5 \%$ y el menos practicado es el principio de autonomía con $28.5 \%$. asimismo un $17.2 \%$ de los profesionales de enfermería no practica los principios de No maleficencia y autonomía.

\section{REFERENCIAS BIBLIOGRÁFICAS}

1. Amaro, M. (2004). Toma de decisiones éticas aplicada a la especialidad de Enfermería. Revista Cubana de Enfermería v.23 n.3. Cuba: Ciudad de la Habana. Recuperado de: file:///H:/investigacion/bibliografia/amaro $\% 202004 b \% 20$ toma $\% 20$ de\%20decisiones\%20eticas.

2. Amaro, M., Marrero, A., Valencia, M., Blanca, S., \& Moynelo, H. (1996). Principios básicos de la bioética. Revista Cubana de Enfermería v.12 n.1 Ciudad de la Habana. ISSN 0864-0319. Recuperado de: http:// bvs.sld.cu/revistas/enf/vol12_1_96/enf06196.htm

3. Baltazar, C., \& García, R. (2009). Nivel de conocimientos bioéticos en el personal de enfermería de un hospital del sureste de Veracruz. Tesis para optar el título de licenciado en enfermería, Facultad de enfermería, Universidad Veracruzana, México.

4. Bermejo, J. (1999). Salir de la noche: por una enfermera humanizada. Barcelona: Ed. Sal terrae. ISBN: 84-293-1317-6.

5. Burkhardt, M., \& Nathaniel, A. (2002). Ethics and Issues in Contemporary Nursing. $2^{\circ}$ Ed. United States of America: Shelley Esposito. Pags. 20- 60, 304-318. ISBN: 0-7668-3629-0.

6. Colegio de Enfermeros del Perú (2005). Código de Ética y Deontología. Lima. Perú. 
7. Colliere, M. (1993). Promover la vida: de la práctica de las mujeres cuidadoras a los cuidados de enfermería. Madrid: Interamericana Mc Graw Hill.

8. Durand, M. (2004). Enfermería, desarrollo teórico e investigativo. Bogotá: unibiblos.

9. García, A. (2007). Competencia y desempeño del personal de enfermería en relación a los principios bioéticos en la Atención Primaria de Salud del personal enfermero, perteneciente al Policlínico Universitario Dr. "Francisco Figueroa Veliz. Cuba. Recuperado de http://www.monografias.com/ trabajos81/competencia-desempeno-enfermeria/ competencia-desempeno-enfermeria2.shtml

10. Gutiérrez, E., \& Páez, C. (2003). Nivel de conocimientos sobre aspectos ético-legales del personal de enfermería que labora en la unidad de emergencia del Hospital Central Universitario: Dr. Antonio María Pineda. Tesis para optar el título profesional de licenciada en enfermería. Facultad de Medicina. Universidad Centro Occidental Lisandro Alvarado. Barquisimeto, Venezuela. Recuperado de: http://bibmed.ucla.edu.ve/cgiwin/be_alex.exe?Ac ceso $=$ T070000059807/0\&Nombrebd=bmucla

11. Hernández, R., Fernández, C., \& Baptista, M. (2010). Metodología de la investigación. 5ta Edición. México: Mc Graw Hill.

12. Luna, L., Valiente, M., Espinosa, E. \& Goderich, J. (2004). Cumplimiento de los principios fundamentales de la bioética por el personal de enfermería en el área de cirugía del Hospital clínico-quirúrgico: Dr. Ambrosio Grillo Portuondo, de Santiago de Cuba. Revista Cubana de Enfermería v20. n.2. Cuba: Ciudad de la Habana. Recuperado de: http://www.bvs.sld.cu/revistas/enf/vol20_2_04/ enf09204.htm

13. Miscán, A., \& Salazar, E. (2009). Autonomy and not balefulness: Ethic experience in the clinical practice. Revista de Enfermería Herediana. v2. n.1. pág. 47-51. Recuperado de: http://www.upch. edu.pe/faenf/revfae/ART7_MISCAN.pdf.

14. Ospina, B., De Jesús, J., Aristizábal, C., \& Ramírez, M. (2003). La escala de Likert en la valoración de los conocimientos y las actitudes de los profesionales de enfermería en el cuidado de la salud. Investigación y Educación en Enfermería - Medellín, marzo, Vol. 23, No 1. Antioquía. Recuperado de:

http://aprendeenlinea.udea.edu.co/revistas/index.
php/iee/article/viewFile/2949/263

15. Ortega, C., y Suárez, M. (2009). Manual de evaluación de la calidad del servicio de enfermería. 2da edición. Argentina: Médica panamericana.

16. Pérez, A. (1999). Problemas éticos en la atención de urgencias. Descripción de experiencias. Revista Cubana de Enfermería. V15. n.2. Cuba: Ciudad de la Habana. Recuperado de: http://bvs.sld.cu/revistas/enf/vol15_2_99/enf14299.pdf

17. Poblete, M., y Valenzuela, S. (2007). Humanized care: a challenge for nursing in the hospitals services. Acta Paul Enfermeria. Vol.20. n ${ }^{\circ}$ 4. Recuperado de http://www.scielo.br/pdf/ape/v20n4/18.pdf

18. Porra, J., Díaz, I., y Cordero, G. (2001). Conocimientos y aplicación de los principios éticos y bioéticos en el proceso de atención de enfermería. Revista Cubana de Enfermería. V17. n.2. Cuba: Ciudad de la Habana. Recuperado de http://bvs. sld.cu/revistas/enf/vol17_2_01/enf11201.pdf

19. Remuzgo, A. (2003). Nivel de satisfacción del paciente adulto mayor respecto a los cuidados que recibe de las enfermeras (os) en el servicio de Gritaría del 120

20. Rodríguez, O. (2003). Exploración y aplicación de conocimientos bioéticos en el personal de enfermería. Revista Cubana de Enfermería. V19. n.3. Cuba: Ciudad de la Habana. Recuperado de http:// bvs.sld.cu/revistas/enf/vol19_3_03/enf10303.pdf

21. Santos, A., Peña, M. (1998). Humanización de la atención sanitaria; retos y perspectivas, Primera Parte: La Deshumanización en la Práctica Sanitaria Actual. http://www.cbioetica.org/revista/42/421218.pdf

22. Taboada, P. (1998). Ética clínica: principios básicos y modelo de análisis. Recuperado de http://escuela.med.puc.cl/paginas/publicaciones/ boletin/html/etica/etica02.pdf

23. Yagüe, A., García, M. (2004). Actitudes de los profesionales de enfermería ante los pacientes terminales. Tesis de posgrado, diplomado en enfermería. Recuperado de: http://www.uclm.es/ab/ enfermeria/revista/numero\%204/terminales4.htm

24. Waldow, R. (2004,2008). Cuidar: expresión humanizadora. Reimpresión. Brasil: palabra. 